\title{
Hepatocyte growth factor renders BRAF mutant human melanoma cell lines resistant to PLX4032 by downregulating the pro-apoptotic BH3-only proteins PUMA and BIM
}

\author{
Leona Rohrbeck ${ }^{1,2}$, Jia-Nan Gong ${ }^{1,2}$, Erinna F Lee ${ }^{1,2,3,4,5}$, Andrew J Kueh ${ }^{1,2}$, Andreas Behren ${ }^{4,5}$, Lin Tai ${ }^{1}$, Guillaume Lessene ${ }^{1,2}$, \\ David CS Huang ${ }^{1,2}$, Walter D Fairlie ${ }^{1,2,3,4,5}$, Andreas Strasser ${ }^{1,2}$ and Marco J Herold ${ }^{\star, 1,2}$
}

\begin{abstract}
A large proportion of melanomas harbour the activating $\mathrm{BRAF}^{\mathrm{V} 600 \mathrm{E}}$ mutation that renders these cells dependent on MAPK signalling for their survival. Although the highly specific and clinically approved BRAF ${ }^{\mathrm{V} 600 \mathrm{E}}$ kinase inhibitor, PLX4032, induces apoptosis of melanoma cells bearing this mutation, the underlying molecular mechanisms are not fully understood. Here, we reveal that PLX4032-induced apoptosis depends on the induction of the pro-apoptotic BH3-only protein PUMA with a minor contribution of its relative BIM. Apoptosis could be significantly augmented when PLX4032 was combined with an inhibitor of the pro-survival protein BCL-XL, whereas neutralization of the pro-survival family member BCL-2 caused no additional cell death. Although the initial response to PLX4032 in melanoma patients is very potent, resistance to the drug eventually develops and relapse occurs. Several factors can cause melanoma cells to develop resistance to PLX4032; one of them is the activation of the receptor tyrosine kinase CMET on melanoma cells by its ligand, hepatocyte growth factor (HGF), provided by the tumour microenvironment or the cancer cells themselves. We found that HGF mediates resistance of CMET-expressing BRAF mutant melanoma cells to PLX4032-induced apoptosis through downregulation of PUMA and BIM rather than by increasing the expression of pro-survival BCL-2-like proteins. These results suggest that resistance to PLX4032 may be overcome by specifically increasing the levels of PUMA and BIM in melanoma cells through alternative signalling cascades or by blocking pro-survival BCL-2 family members with suitable $\mathrm{BH} 3$ mimetic compounds.
\end{abstract}

Cell Death and Differentiation (2016) 23, 2054-2062; doi:10.1038/cdd.2016.96; published online 30 September 2016

A large fraction of melanomas harbours the BRAF ${ }^{\mathrm{V} 600 E}$ mutation, which accounts for $70-90 \%$ of BRAF mutations that are found in melanomas. This T1799A transversion results in a 500-fold increase in BRAF kinase activity, thereby driving MAPK signalling independent of external stimuli. ${ }^{1,2}$ Activation of the MAPK pathway promotes proliferation and survival of cells through ERK1/2-mediated control of downstream target genes, including the negative regulation of the pro-apoptotic BCL-2 family member BIM. ${ }^{3,4}$

PLX4032 (Vemurafenib) is a clinically approved inhibitor specific for BRAF ${ }^{\mathrm{V} 600 E}$. It causes cell cycle arrest and apoptosis in BRAF mutant melanomas but not in those expressing wild-type BRAF. ${ }^{5}$ Previous studies demonstrated that apoptosis of BRAF ${ }^{\mathrm{V} 600 \mathrm{E}}$ melanoma cells triggered by MEK1/2 inhibitors or PLX4032 was partially dependent on $\mathrm{BIM}$, as RNA interference mediated knockdown of BIM significantly reduced cell killing, although it did not abolish it. $^{6,7}$ This suggests that other pro-apoptotic BH3-only members of the BCL-2 family are likely to co-operate with BIM in PLX4032-induced apoptosis of these melanomas.
The BCL-2 protein family can be subdivided into three groups: the pro-survival proteins (BCL-2, BCL-XL, MCL-1, $B F L-1 / A 1$ and $B C L-W)$, the BH3-only proteins (BIM, PUMA, NOXA, BID, BAD, HRK, BMF, BAD and BIK) and the multi-BH domain containing pro-apoptotic proteins, BAX, BAK and possibly BOK, which cause mitochondrial outer membrane permeabilization and thereby unleash cellular demolition by the caspases. $^{8,9}$ The BH3-only proteins initiate apoptosis signalling either through direct activation of BAX/BAK or indirectly by binding to the pro-survival BCL-2-like proteins, thereby releasing $\mathrm{BAX/BAK}$ from their restraint by their pro-survival relatives. ${ }^{10}$ Hence, inhibition of pro-survival BCL-2 family members by small molecule $\mathrm{BH} 3$ mimetics can initiate apoptosis signalling. $\mathrm{ABT}-737$ is a $\mathrm{BH} 3$ mimetic that binds with high affinity to BCL-2, BCL-XL and BCL-W, but not to MCL-1 or $\mathrm{BFL}-1 .{ }^{11}$ In cancers that are driven by aberrant expression of oncogenic kinases, potent synergy between ABT-737 and inhibitors of these kinases was observed. ${ }^{12,13}$ Although it has been reported that ABT-737 synergizes with PLX4032 or a MEK inhibitor in the killing of BRAF mutant melanoma cells, ${ }^{6,7,14}$ for

\footnotetext{
${ }^{1}$ The Walter and Eliza Hall Institute of Medical Research, Parkville, Victoria, Australia; ${ }^{2}$ Department of Medical Biology, University of Melbourne, Parkville, Victoria, Australia; ${ }^{3}$ Department of Chemistry and Physics, La Trobe Institute for Molecular Science, La Trobe University, Bundoora, Victoria, Australia; ${ }^{4}$ Olivia Newton-John Cancer Research Institute, Heidelberg, Victoria, Australia and ${ }^{5}$ School of Cancer Medicine, La Trobe University, Melbourne, Victoria, Australia

${ }^{*}$ Corresponding author: MJ Herold, Department of Medical Biology, The Walter and Eliza Hall Institute of Medical Research, The University of Melbourne, $1 \mathrm{G}$ Royal Parade, Parkville, VIC 3052, Australia. Tel: +61 39345 2555; Fax: +61 39347 0852; E-mail: herold@wehi.edu.au

Abbreviations: BCL2, b-cell lymphoma 2; BCL-XL, apoptosis regulator BCL-XL; BH3, bcl2-homology domain 3; BIM, bcl2-interacting mediator of cell death; ERK, extracellular signal-regulated kinase; HGF, hepatocyte growth factor; MAPK, mitogen-activated protein kinase; MOMP, mitochondrial outer membrane permabilisation; PUMA, P53 upregulated modulator of apoptosis

Received 12.1.16; revised 28.7.16; accepted 02.8.16; Edited by G Melino; published online 30 September 2016
} 

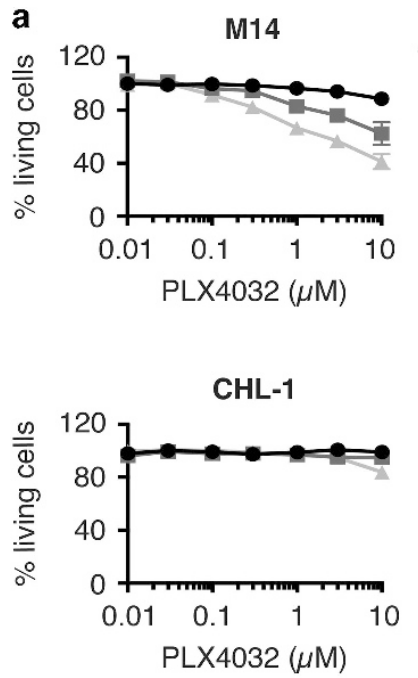
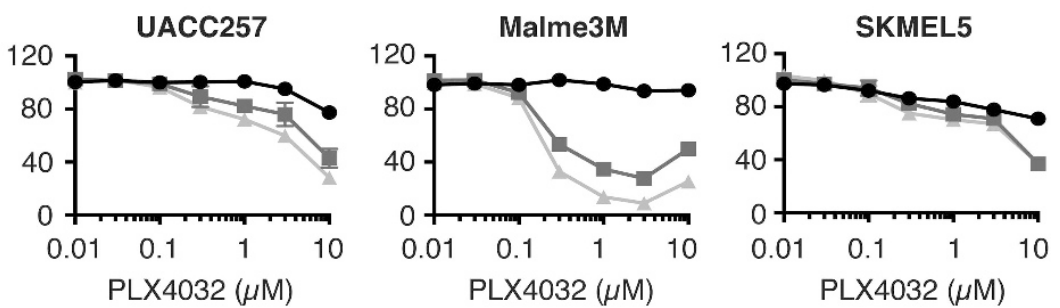

b

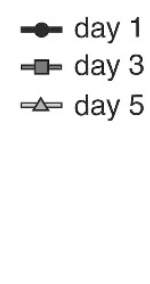

Figure $1 \mathrm{PLX} 4032$ causes apoptosis of BRAF ${ }^{\mathrm{V} 600 \mathrm{E}}$ mutant melanoma cell lines, and this is accompanied by a decrease in phosphorylated (i.e., activated) ERK ( $\mathrm{pERK}$ ) levels. (a) BRAF ${ }^{\mathrm{V} 600 \mathrm{E}}$ mutant and BRAF ${ }^{\mathrm{WT}}$ melanoma cell lines were treated with DMSO (vehicle) or PLX4032 $(0.01,0.03,0.1,0.3,1,3,10 \mu \mathrm{M})$ for the indicated times and cell survival was analyzed by flow cytometry. Living cells were identified as being negative for Annexin $V$ and propidium iodide staining. The data are derived from three independent experimental replicates, each comprising the average of three technical replicates and the data are presented as mean \pm S.E.M. (b) M14, UACC257, Malme3M, SKMEL5 (all mutant $\mathrm{BRAF}^{\mathrm{V} 600 \mathrm{E}}$ ) and $\mathrm{CHL}-1$ (BRAF ${ }^{\mathrm{WT}}$ ) were treated with DMSO (vehicle; -) or PLX4032 (+; at $\left.3 \mu \mathrm{M}\right)$ in the presence of the pan-caspase inhibitor Q-VD-OPh for $24 \mathrm{~h}$ before western blot analysis to detect PERK and total ERK protein levels. Probing for HSP70 was used as a loading control

designing optimal combination therapies, it is crucial to understand which of the pro-survival family members targeted by this $\mathrm{BH} 3$ mimetic compound is essential for the sustained growth of melanoma cells.

One feature melanocytes must acquire during their transformation to malignant melanoma is growth autonomy. Cell proliferation is normally dependent on growth factor receptormediated signalling. Not surprisingly, many cancers express high levels of growth factor receptors and sometimes even their ligands. Alternatively, the ligands can be provided by surrounding stromal cells. Accordingly, it was recently suggested that autonomous growth factor receptor-mediated signalling renders melanoma cells resistant to PLX4032 and therefore causes patients to relapse. ${ }^{15}$ Specifically, it was reported that secretion of hepatocyte growth factor (HGF) from the tumour microenvironment and consequent activation of its receptor tyrosine kinase, cMET, which is expressed on a subset of the melanoma cells, promotes outgrowth of PLX4032-resistant cancer cells. ${ }^{16,17}$ In this study, we examined the importance of the intrinsic apoptotic pathway in PLX4032-induced killing of melanoma cells and its role in HGF/cMET signalling-driven resistance to this drug. Understanding these mechanisms will be crucial for the identification of novel therapeutic targets in BRAF $^{\mathrm{V} 600 E}$ melanomas and possibly other cancers that express cMET.

\section{Results}

Human melanoma cells with the BRAF ${ }^{\mathrm{V} 600 \mathrm{E}}$ mutation are killed by PLX4032. PLX4032 is a small molecule compound designed to inhibit the mutant BRAF ${ }^{\mathrm{V} 600 \mathrm{E}}$ kinase, which drives activation of downstream kinases in the MAPK pathway, such as ERK1/2, thereby promoting proliferation and survival of melanoma cells. To investigate the molecular events by which
PLX4032 mediates melanoma cell killing, we assembled a panel of well-characterized human melanoma cell lines and treated them with titrated doses of PLX4032 to determine their sensitivity to this agent. We found that PLX4032 induced significant dose-dependent apoptosis over a time course of 5 days in all BRAF mutant melanoma cell lines (M14, UACC257, Malme3M, SKMEL5 and UACC62), whereas the BRAF $^{\mathrm{WT}}$ cells $(\mathrm{CHL}-1)$ were unaffected (Figure 1a and Supplementary Figure 1a). As expected and previously reported, ${ }^{5}$ treatment with $\mathrm{PLX} 4032$ reduced phosphorylation of ERK1/2 in the BRAF ${ }^{\mathrm{V} 600 E}$ mutant melanoma cell lines, whereas ERK phosphorylation was paradoxically increased in the BRAF ${ }^{W T}$ cell line (Figure 1b). The sensitivity of the different melanoma cell lines to PLX4032-induced apoptosis was mirrored by the extent of repression of ERK1/2 activity, as reflected by reduction of ERK1/2 phosphorylation.

PUMA and to a lesser extent BIM are critical for PLX4032induced apoptosis of BRAF ${ }^{\mathrm{V} 600 E}$ mutant melanoma cells. The pro-apoptotic BH3-only protein BIM has a crucial role in PLX4032-induced killing. ${ }^{6}$ However, knockdown of BIM was not sufficient to completely abrogate PLX4032-induced cell death, ${ }^{7,14}$ which suggests that other pro-apoptotic BCL-2 family members may also contribute. To this end, we investigated changes in the expression of $\mathrm{BH}$-only proteins in melanoma cells upon treatment with PLX4032.

As expected, we observed strong upregulation of BIM in all BRAF mutant cells after $24 \mathrm{~h}$ of treatment (Figure 2a and Supplementary Figure 1b). Interestingly, we additionally observed significant increase in PUMA protein levels in all BRAF mutant cells but not in the BRAF wild-type (WT) melanoma cell line (Figure $2 \mathrm{a}$ and Supplementary Figure 1b). No consistent changes in BMF and NOXA levels were observed. Although PUMA has been reported as a transcriptional target of the tumour-suppressor $\mathrm{p} 53,^{18,19}$ 


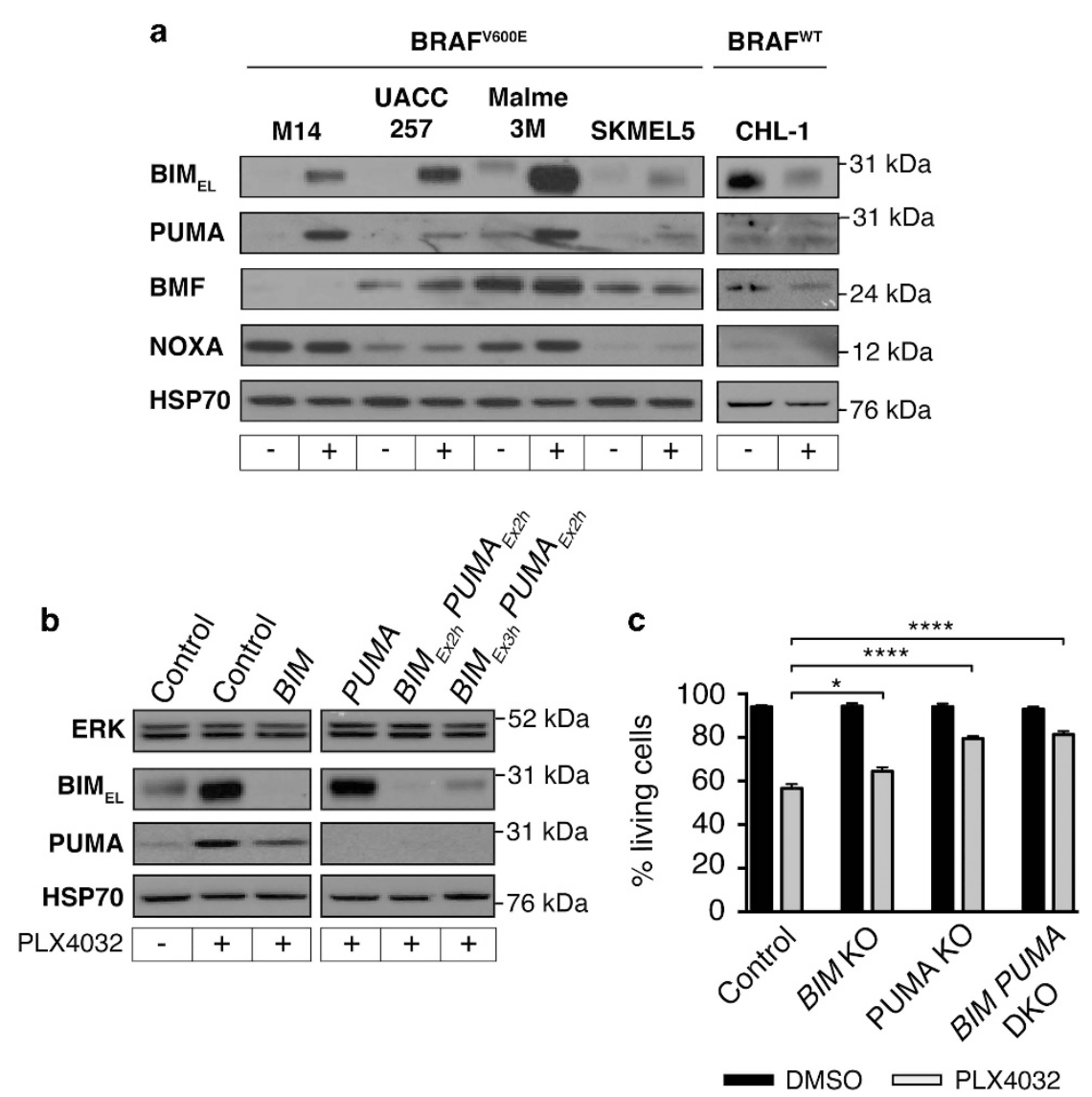

Figure 2 PLX4032 mediates apoptosis of BRAF mutant melanoma cell lines through upregulation of the two BH3-only proteins, PUMA and BIM. (a) BRAF ${ }^{\mathrm{V} 600 \mathrm{E}}$ mutant and $\mathrm{BRAF}^{\mathrm{WT}}$ melanoma cell lines were treated with DMSO (vehicle; -) or PLX4032 (+; at $\left.3 \mu \mathrm{M}\right)$ in the presence of the pan-caspase inhibitor Q-VD-OPh for $24 \mathrm{~h}$ and western blot analysis was performed to measure the expression levels of the indicated BH3-only proteins. Probing for HSP70 was used as a loading control. (b) M14 BRAF ${ }^{\mathrm{V} 600 \mathrm{E}}$ mutant cells were transduced with a Cas9 expression vector and the indicated human BIM and/or human PUMA sgRNAs, targeting the genes either in exon 2 (Ex2h) or 3 (Ex3h). Control cells were transduced with a sgRNA targeting mouse Bim. M14 control or knockout cell lines were treated with DMSO (vehicle; -) or PLX4032 (+, at $3 \mu \mathrm{M}$ ) for $24 \mathrm{~h}$ in the presence of Q-VD-OPh, and BIM and PUMA protein levels were determined by western blot analysis. Probing for ERK and HSP70 was used as a loading control. (c) Cell lines from panel (b) were treated with DMSO (vehicle; black bar) or PLX4032 (grey bar, at $3 \mu \mathrm{M}$ ) for 5 days and cell survival was measured by flow cytometric analysis. Living cells were identified as those negative for Annexin V. The contribution of pooled sgRNAs is illustrated. The data are derived from three independent experimental replicates, each comprising the average of three technical replicates. The graph demonstrates the survival advantage of knockout cells relative to control cells. The data are presented as mean \pm S.E.M.; ${ }^{*} P<0.05$, ${ }^{* \star \star \star} P<0.0001$

PLX4032-induced upregulation of PUMA (and also BIM) was independent of p53 (Supplementary Figures 2a-c).

To determine the functional relevance of PUMA upregulation in PLX4032-mediated killing of BRAF mutant melanoma cells, we generated BIM and PUMA single (M14, UACC62 and UACC257) and double knockout cell lines (M14 and UACC62), using CRISPR/Cas9. BIM and PUMA were each targeted with two independent sgRNAs against exon 2 or exon 3 of the genes: BIMEx2h, BIMEx3h, PUMAEx2h or PUMAEx3h. Efficient targeting of BIM and/or PUMA with the sgRNAs was already validated previously. ${ }^{20,21}$ Knockout of BIM and PUMA in both, single and double knockout cell lines, was verified, with these proteins being either undetectable or at least markedly reduced in all cell lines after treatment with PLX4032 (Figure 2b and Supplementary Figure 1b). As expected, PLX4032-induced upregulation of BIM and PUMA occurred normally in the control sgRNA transduced cell lines. Notably, the CRISPR/Cas9 knockout of BIM and PUMA was performed on a bulk cell population, rather than on single-cell clones, which excludes selection for potential unrelated mutations that could affect the response to PLX4032 during the single-cell cloning process. Importantly, PLX4032 treatment was still able to downregulate ERK activity in BIM and/or PUMA-deficient M14 and UACC257 cells, as reflected by the reduction in the levels of phosphorylated ERK ( $p E R K$ ) (Supplementary Figures $1 \mathrm{~b}$ and c). Owing to the relatively minor impact of PLX4032 on the UACC62 cells, we were unable to see any marked changes in pERK in these cells (Supplementary Figure 1b). However, as BIM or PUMA were still upregulated in response to PLX4032 in the PUMA- or BIMdeficient variants, respectively, we are confident that the cellular pathways induced by mutant BRAF inhibition must be largely intact in these cells.

To examine the relative importance of PUMA and BIM in PLX4032-induced apoptosis in BRAF mutant melanomas, the single and double knockout cell lines were treated with PLX4032 and the extent of cell killing determined. Consistent with previous studies, ${ }^{7,14}$ we found that targeting BIM significantly reduced PLX4032-induced apoptosis, albeit to a relatively minor extent (Figure $2 \mathrm{c}$ and Supplementary Figure 1d). Interestingly, PUMA deficiency resulted in a more prominent protection from PLX4032-induced cell death and cell 


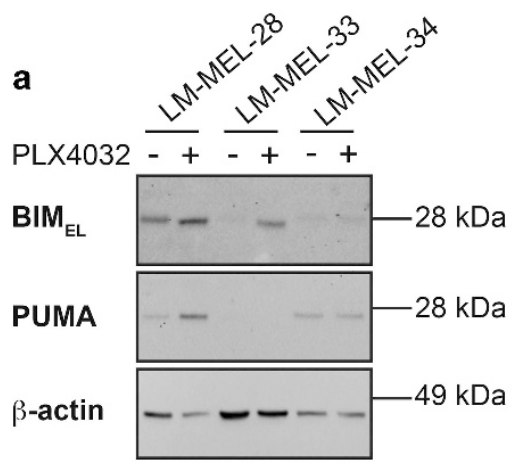

b
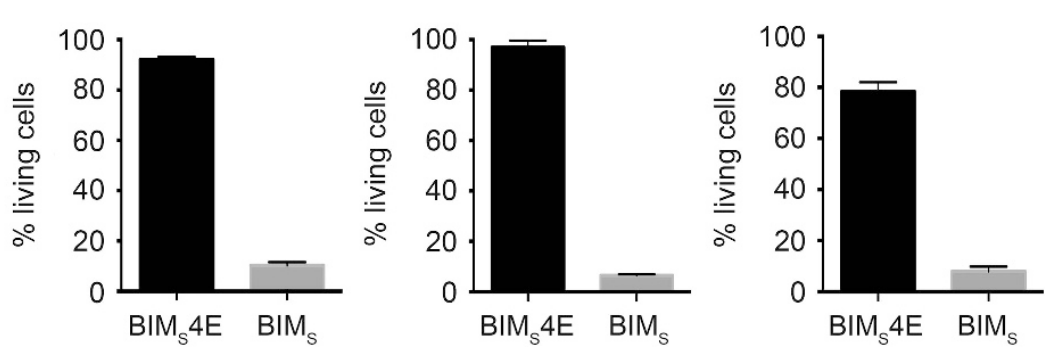

Figure 3 Patient-derived (early passage) human mutant BRAF melanoma cells upregulate BIM and PUMA in response to PLX4032 treatment. (a) Patient-derived cell lines ${ }^{22}$ LM-MEL-28 (BRAF mt), -33 (BRAF mt), -34 (BRAF wt) were treated with DMSO (vehicle; -) or PLX4032 (+; at $3 \mu \mathrm{M}$ ) in the presence of the pan-caspase inhibitor Q-VD-OPh for $24 \mathrm{~h}$ before western blot analysis to detect the indicated BH3-only proteins. Probing with an antibody to $\beta$-actin was used as a loading control. (b) Patient-derived cell lines were transduced with lentiviruses to enable expression of $\mathrm{BIM}_{\mathrm{S}}$ or an inert mutant $(\mathrm{BIM} 4 \mathrm{E})$. Ligand expression was induced following addition of doxycycline and cell viability determined 3 days later by flow cytometric analysis. Living cells were identified as those negative for Annexin V. The data are derived from three independent experimental replicates, each comprising the average of three technical replicates and are presented as mean \pm S.E.M.

survival was even further enhanced when both, BIM and PUMA, were deleted (Figure $2 \mathrm{c}$ and Supplementary Figure 1d).

Primary human BRAF mutant melanoma cells upregulate PUMA and BIM upon treatment with PLX4032. In order to test the apoptotic response of low passage patient-derived melanoma cell lines, ${ }^{22}$ we treated the BRAF ${ }^{\mathrm{V} 600 E}$ lines LMMEL-28 and LM-MEL-33 and, as a control, the BRAF ${ }^{\mathrm{WT}}$ line, LM-MEL-34, with PLX4032. As expected, we observed significant killing of the two lines bearing mutant BRAF, whereas the BRAF ${ }^{\mathrm{WT}}$ cells were not adversely affected (Supplementary Figure 3). Although no upregulation of proapoptotic $\mathrm{BH}$-only proteins was observed in the BRAFWT LM-MEL-34 cells, BIM was markedly upregulated in both BRAF $^{\mathrm{V} 600 E}$ melanoma lines. However, PUMA was only upregulated in LM-MEL-28, but not in LM-MEL-33 cells (Figure 3a). Interestingly, the LM-MEL-28 cells, were more efficiently killed by PLX4032 compared with the LM-MEL33 cells (Supplementary Figure 3 ). This agrees with our previous observation that PUMA is the main killer protein in PLX4032treated mutant BRAF melanoma cells, as strong upregulation of BIM was seen in both LM-MEL-28 and LM-MEL-33 cells (Figure 3a), whereas only the LM-MEL-28 cells also showed marked upregulation of PUMA. Notably, when BIMS was overexpressed using a doxycycline-inducible lentiviral vector system, ${ }^{23}$ LM-MEL-28 and LM-MEL-33 were killed with similar efficiencies. Enforced expression of the inert BIMS4E mutant (negative control), ${ }^{24}$ which does not bind to any pro-survival BCL-2 family members or BAX/BAK, had no impact (Figure $3 b$ ). This confirms that all of these cell lines contain the functional machinery, capable of activating the intrinsic apoptotic pathway. Collectively, these findings demonstrate that the pro-apoptotic BH3-only proteins PUMA and BIM are essential for PLX4032-induced killing of early passage BRAF $^{\mathrm{V} 600 \mathrm{E}}$ expressing patient-derived melanoma cells, similar to that observed in established cell lines (Figure 2).

BCL-XL inhibition significantly increases PLX4032induced killing of BRAF mutant melanoma cells. We have shown that PUMA and BIM are the main mediators of
PLX4032-induced apoptosis in BRAF ${ }^{\mathrm{V} 600 E}$ melanoma cells. Further insight into the key regulators of cell death in melanomas may facilitate the development of combination therapies to enhance the efficacy of PLX4032. Potential therapeutic targets of particular interest are the endogenous antagonists of PUMA and BIM, the BCL-2-like pro-survival proteins. BCL-2, and its homologues, BCL-XL, MCL-1 and BFL-1, have all been reported to contribute to different stages of melanoma development. ${ }^{25-27}$ Western blot analysis identified BCL-2, BCL-XL and MCL-1 at readily detectable levels in the BRAF ${ }^{V 60 E}$ melanoma cell lines tested and their expression levels did not significantly change when these cells were treated with PLX4032, except for the Malme3M cells, in which a minor decrease of all pro-survival BCL-2 proteins was observed (Figure 4a). BFL-1 protein levels could not be determined, as there are no reliable antibodies available.

We next investigated, which of the pro-survival BCL-2 protein/s is/are critical for the sustained survival and growth of mutant BRAF-driven melanoma cells, both under normal conditions and following PLX4032 treatment. Previous studies have demonstrated that PLX4032-induced killing of melanoma cells can be augmented by co-treatment with ABT-737, a $\mathrm{BH} 3$ mimetic that inhibits BCL-2, BCL-XL and BCL-W. ${ }^{7,11,14}$ We sought to extend these findings by determining which of these three pro-survival BCL-2 family member(s) targeted by ABT-737 is/are critical for melanoma cell survival. The BRAF mutant melanoma lines were treated with the $B C L-2$ selective inhibitor, ABT-199, ${ }^{28}$ or the BCL-XL-specific compound, A$1155463,{ }^{29}$ either alone or in combination with PLX4032. As a control, the cells were also treated with ABT-737 or ABT-737 plus PLX4032. As reported, ${ }^{7,14}$ pronounced induction of apoptosis was seen in BRAF mutant melanoma cell lines when they were exposed to a combination of PLX4032 plus ABT-737 (Figure 4b). Inhibition of BCL-XL in combination with PLX4032 led to a similar extent of apoptosis in the BRAF mutant melanoma cells, whereas the combination of BCL-2 inhibition with PLX4032 had much lower impact. Notably, neither ABT-737, ABT-199 nor A-1155463 induced significant cell death as single agents in any of the melanoma cell lines tested (Figure $4 \mathrm{~b}$ ). To examine the role of $B C L-X L$ in the 


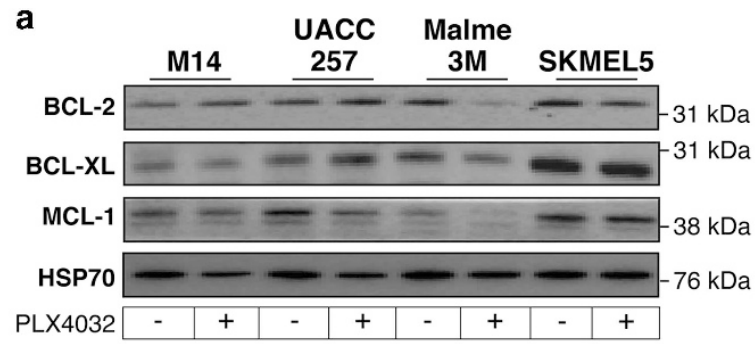

b

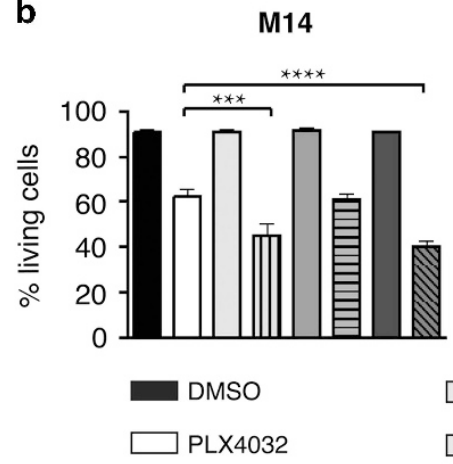

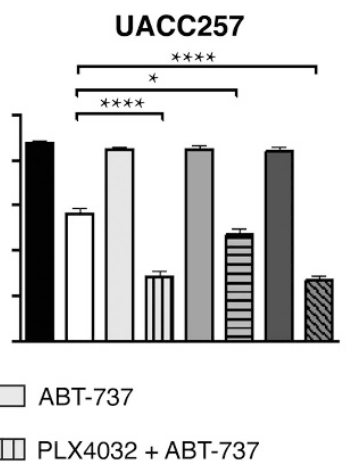

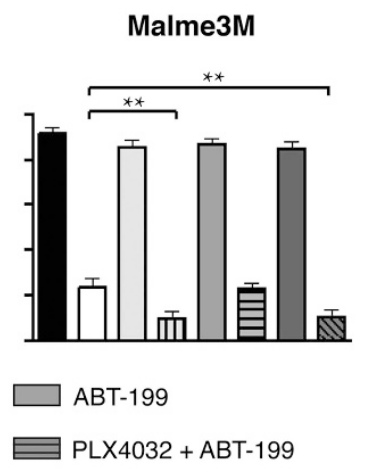

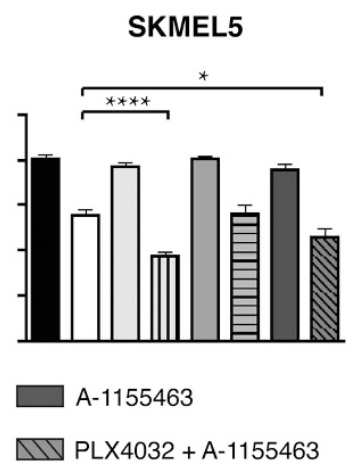

Figure 4 Inhibition of $\mathrm{BCL}-\mathrm{XL}$ but not inhibition of $\mathrm{BCL}-2$ cooperates with $\mathrm{PLX} 4032$ in the killing of $\mathrm{BRAF}{ }^{\mathrm{V} 600 \mathrm{E}}$ mutant melanoma cell lines. (a) M14, UACC257, Malme3M and SKMEL5 BRAF ${ }^{\mathrm{V} 600 \mathrm{E}}$ cell lines were treated with DMSO (vehicle; -) or PLX4032 (+; at $\left.3 \mu \mathrm{M}\right)$ in the presence of the pan-caspase inhibitor Q-VD-OPh for $24 \mathrm{~h}$ before western blot analysis to detect the indicated pro-survival BCL-2-like proteins. Probing with an antibody to HSP70 was used as a loading control. (b) BRAF ${ }^{\mathrm{V} 600 \mathrm{E}}$ mutant melanoma cell lines from panel (a) were treated with DMSO, PLX4032 (3 $\mu \mathrm{M})$ and/or (c) ABT-737 (1 $\mu \mathrm{M}$; inhibitor of BCL-2, BCL-XL and BCL-W), ABT-199 (1 $\mu$ M; inhibitor of BCL-2) or ABT-1155463 $(1 \mu \mathrm{M}$; inhibitor of $\mathrm{BCL}-\mathrm{XL})$. Cell survival was assessed after 5 days of treatment by flow cytometric analysis. Living cells were identified as those negative for Annexin $\mathrm{V}$ and propidium iodide. The data are derived from three independent experimental replicates, each comprising the average of three technical replicates and are presented as mean \pm S.E.M.; ${ }^{\star} P<0.05,{ }^{\star \star} P<0.01,{ }^{* \star \star} P<0.001,{ }^{\star \star * \star} P<0.0001$

survival of the low passage patient-derived melanoma lines, cells were treated with a combination of PLX4032 plus the BCL-XL inhibitor, A-1155463. These compounds potently cooperated in the killing of the LM-MEL-33 cells, but only showed additive effects in the LM-MEL-28 cells (Supplementary Figure 3). The latter finding can probably be attributed to the fact that treatment with PLX4032 alone potently kills LM-MEL-28 cells, hence no further increase in killing would be expected in this line. Our results show that $\mathrm{BRAF}^{\mathrm{V} 600 \mathrm{E}}$ melanoma cells rely on BCL-XL but not on BCL-2 or BCL-W for their survival, particularly when treated with PLX4032. In addition, our data reveal that inhibition of BCL-XL by A-1155463 only sensitizes BRAF mutant melanoma cells to apoptosis, and that PLX4032-mediated upregulation of PUMA and BIM is critical for their efficient killing.

HGF-induced resistance of BRAF ${ }^{\mathrm{V} 600 \mathrm{E}}$ melanoma cell lines to PLX4032 is mediated by inhibiting upregulation of PUMA and BIM. Despite a strong initial response of $\mathrm{BRAF}^{\mathrm{V} 600 \mathrm{E}}$ melanoma cells to PLX4032, often resulting in profound tumour regression, relapse of drug-resistant tumours invariably occurs. ${ }^{15}$ Recent studies have implicated HGF/CMET signalling as an important resistance mechanism acquired by BRAF mutant melanoma cells following PLX4032 treatment. ${ }^{16,17} \mathrm{HGF}$ mediates its activity through activation of the CMET receptor tyrosine kinase that is expressed on the surface of human melanoma cell subsets. ${ }^{30}$ We quantified CMET expression on the BRAF ${ }^{\mathrm{V} 600 \mathrm{E}}$ melanoma cells by flow cytometry, classifying them as CMET+ or cMET- (Supple- mentary Figure 4a). As expected, only the CMET+ melanoma cell lines (Malme3M, SKMEL5 and UACC62) became resistant to PLX4032 treatment when co-treated with HGF, whereas the CMET- lines (M14 and UACC257) remained sensitive to this inhibitor of mutant BRAF (Figure $5 \mathrm{a}$ and Supplementary Figure $4 b)$.

We hypothesized that modulation of BCL-2 family member levels is likely to contribute to HGF-mediated resistance to PLX4032. Therefore, we examined the effect of HGF on the expression of pro-survival and pro-apoptotic BCL-2 family members. HGF treatment in the presence or absence of PLX4032 did not alter the mRNA levels of any of the BCL-2 family members tested (Figure $5 b$ ). As expected, at the protein level, HGF had no effect on any of the BCL-2 family members tested in the CMET- cell lines M14 and UACC257 (Figure 5c). Perhaps surprisingly, addition of HGF to PLX4032-treated CMET+ cell lines (Malme3M, SKMEL5 or UACC62) did not elicit changes in the pro-survival proteins, BCL-2, BCL-XL and MCL-1. Instead, we found that HGF-mediated resistance to PLX4032-induced apoptosis by preventing the upregulation of PUMA and BIM (Figure 5c and Supplementary Figure 4c).

HGF-induced resistance to PLX4032 can partially be overcome through BCL-XL inhibition. As BCL-XL inhibition with A-1155463 synergized with PLX4032, we investigated whether this combination could overcome HGF-mediated resistance of BRAF ${ }^{\mathrm{V} 600 E}$ melanoma cells to PLX4032. The CMET+ BRAF mutant cells were treated with PLX4032 in the presence of HGF and A-1155463, or as a control 
a
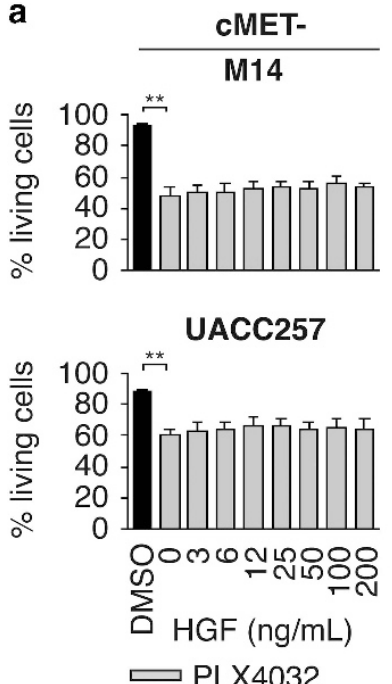

cMET+ Malme3M
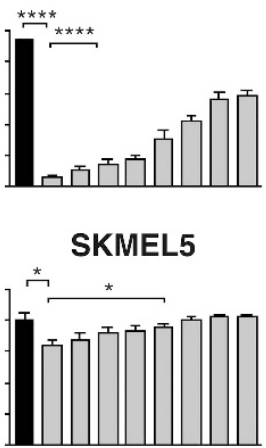

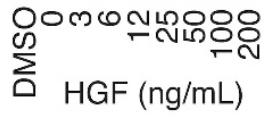

C

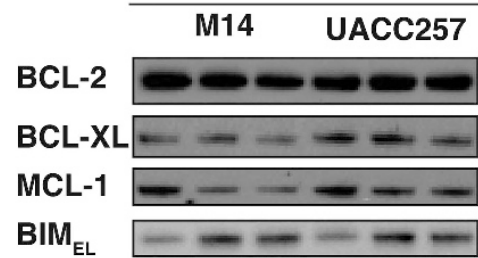

PUMA

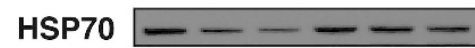

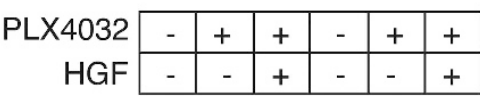

cMET+

Malme3M SKMEL5

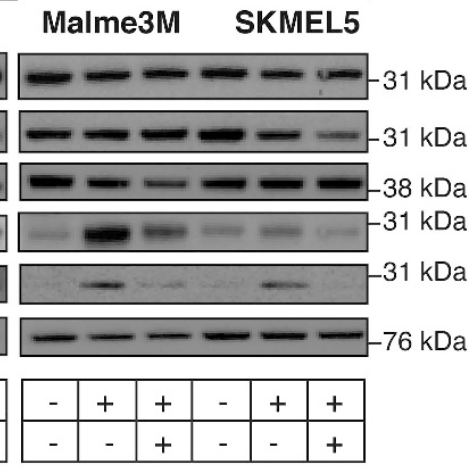

b cMET-
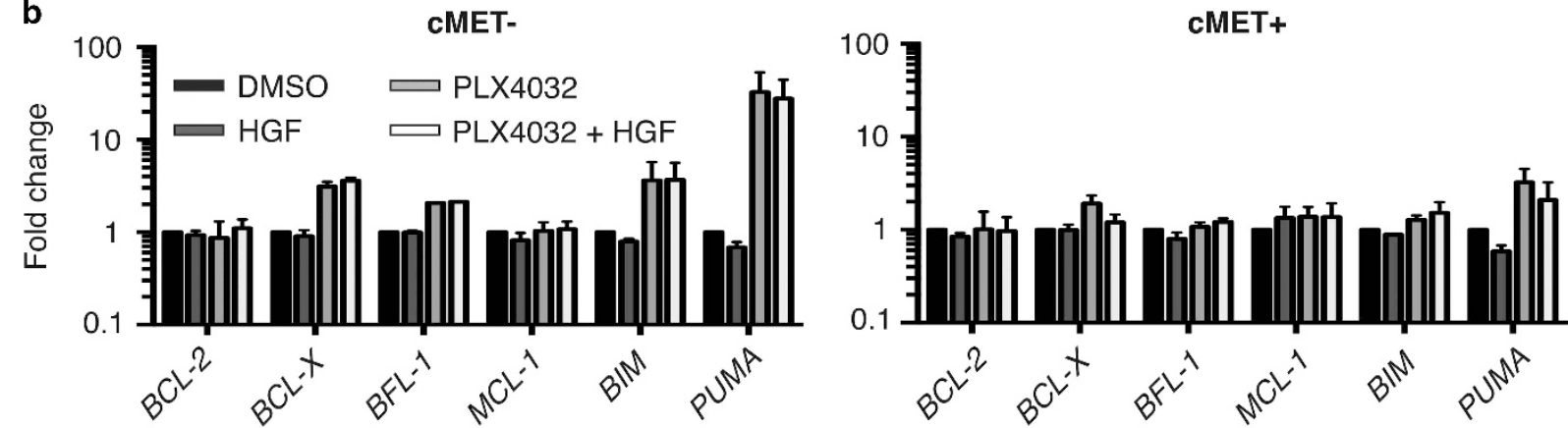

Figure 5 HGF inhibits PLX4032-induced upregulation of PUMA and BIM in CMET+ BRAF mutant melanoma cells and thereby protects them against PLX4032-induced killing. (a) CMET - (M14 and UACC257) and CMET+ (Malme3M and SKMEL5) BRAF ${ }^{\mathrm{V} 00 \mathrm{E}}$ melanoma cell lines were treated with DMSO (vehicle; black bar) or PLX4032 (grey bars, at $3 \mu \mathrm{M}$ ) with the indicated concentrations of $\operatorname{HGF}(0,3,6,12,25,50,100,200 \mathrm{ng} / \mathrm{ml})$ for 5 days and cell survival was assessed by flow cytometry. Living cells were defined as those negative for Annexin V and propidium iodide. The concentration at which HGF causes significant protection from PLX4032-induced killing is indicated. Data are derived from three independent experimental replicates, each comprising the average of three technical replicates and are presented as mean \pm S.E.M.; ${ }^{*} P<0.05,{ }^{* \star} P<0.01,{ }^{* \star * \star} P<0.0001$. (b) $\mathrm{cMET}-(\mathrm{M} 14$ and UACC257) and CMET+ (Malme3M and SKMEL5) BRAF mutant cells were treated with single agents or combinations of DMSO (vehicle), HGF (50 ng/ml) and PLX4032 ( $3 \mu \mathrm{M})$ for $24 \mathrm{~h}$ in the presence of Q-VD-OPh. The mRNA of cells was subjected to qRT-PCR analysis to measure the expression of pro-survival (BCL-2, BCL-X, BFL-1, MCL-1) and proapoptotic (BIM, PUMA) BCL-2 family members. The mRNA expression levels of the different genes were normalized to the levels of Gapdh and the data are presented as fold change relative to DMSO (vehicle)-treated control cells. The data are derived from three independent experimental replicates and are presented as mean \pm S.E.M. (c) Cells were treated as described in panel (b) and analyzed by western blotting for the expression of pro-survival (BCL-2, BCL-XL, MCL-1) and pro-apoptotic (BIM, PUMA) BCL-2 family members. Probing for HSP70 was used as a loading control

with ABT-737 or ABT-199. Co-treatment with A-1155463 (or ABT-737) was able to partially overcome the resistance to PLX4032 conferred by HGF in the Malme3M cells but not in the SKMEL5 cells (Figure 6a). Co-treatment with ABT-199 had no effect. Collectively, these findings suggest that while combination therapy of PLX4032 and A-1155463 can be effective in melanoma, the presence of high levels of HGF, and potentially ligands for other receptor tyrosine kinases or other resistance mechanisms, such as those present in the SKMEL5 cells, will require inclusion of additional drugs to achieve effective combination treatment strategies.

\section{Discussion}

The high incidence of melanomas harbouring activating mutations in the BRAF kinase demonstrates the essential role for MAPK pathway signalling in the development and progression of melanoma. In this study, we investigated the underlying molecular mechanisms of the sensitivity, as well as HGF-mediated resistance to PLX4032-induced apoptosis observed in BRAF ${ }^{\mathrm{V} 600 \mathrm{E}}$ melanoma cell lines. In contrast to previous studies, which solely described a role for BIM in PLX4032-induced apoptosis,, 14 our results show that inhibition of MAPK signalling in human BRAF ${ }^{\mathrm{V} 600 \mathrm{E}}$ melanoma cell lines strongly increased expression of PUMA in addition to BIM. Pertinently, we generated BIM and PUMA single and double knockout cells and showed that PUMA was more important than BIM for PLX4032-induced killing of BRAF mutant lines. PUMA was originally identified as a direct target of the transcription factor p53 (refs 18,19) that is activated in response to many stressors, particularly DNA damage and hypoxia, and is critical for their ability to induce apoptosis. ${ }^{31,32}$ However, PUMA can also be upregulated and induce apoptosis in response to p53-independent apoptotic stimuli, such as cytokine withdrawal or treatment with glucocorticoids. ${ }^{31-33} \mathrm{We}$ generated TP53-deficient BRAF ${ }^{\mathrm{V} 600 \mathrm{E}}$ melanoma cells and 

CMET+
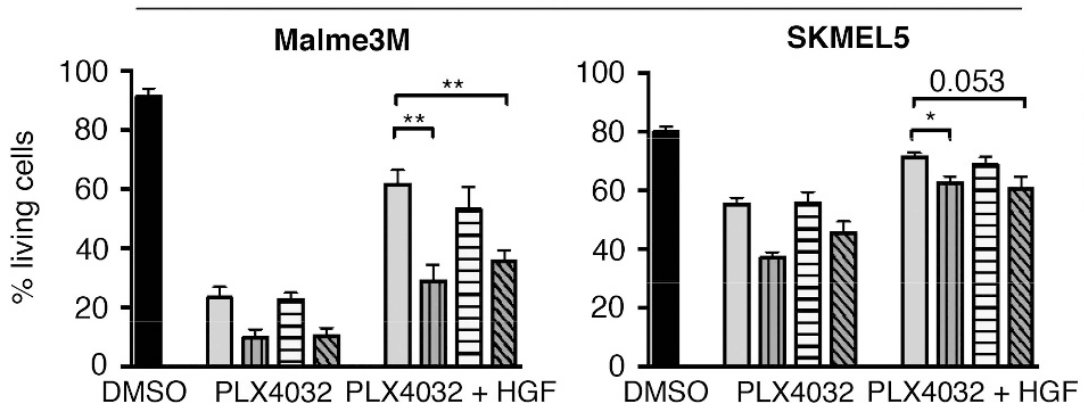

b
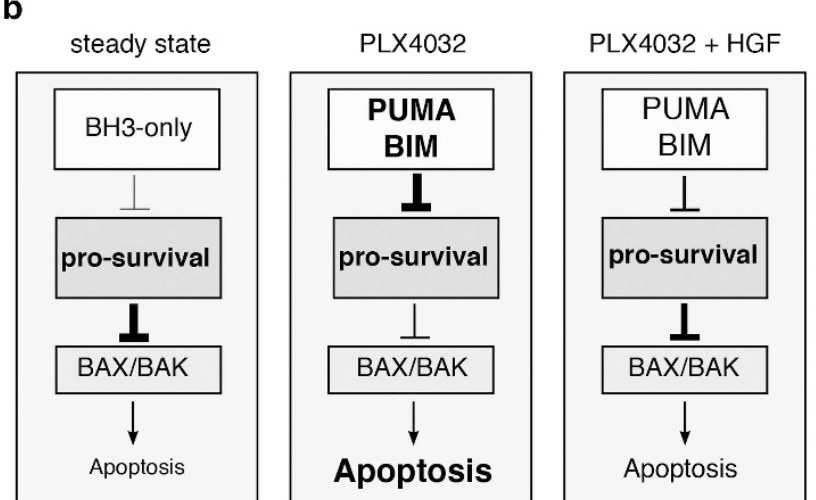

$\mathrm{PLX} 4032+\mathrm{HGF}$ $+\mathrm{A}-1155463$

Figure 6 The combination of PLX4032 and BCL-XL inhibition can partially overcome HGF-mediated resistance of CMET+ BRAF mutant melanoma cells. (a) Malme3M and SKMEL5 cells were treated with DMSO (vehicle), PLX4032 (3 $\mu \mathrm{M})$ alone or in combination with ABT-737 (1 $\mu \mathrm{M})$ (blue bars), ABT-199 (1 $\mu \mathrm{M})$ (green bars), A-1155463 $(1 \mu \mathrm{M})$ (red bars), with or without addition of $\mathrm{HGF}(50 \mathrm{ng} / \mathrm{ml})$ for 5 days and cell survival was assessed by flow cytometry. Living cells were identified as those negative for Annexin V and PI. The data are derived from three independent experimental replicates, each comprising the average of three technical replicates. The data were analyzed using Student's $t$-test and are presented as mean \pm S.E.M.; ${ }^{*} P<0.05,{ }^{* *} P<0.01$. (b) Under steady-state conditions, low levels of pro-apoptotic BH3-only proteins are present in the BRAF mutant melanoma cells. Hence, pro-survival proteins are able to neutralize BAX and BAK to cause survival of the cell. In the presence of PLX4032, PUMA and BIM are getting induced, which leads to sequestration of pro-survival proteins, allowing BAX and BAK to induce apoptosis. In the presence of HGF, PLX4032 mediated upregulation of PUMA and BIM is substantially diminished, and this is the underlying molecular mechanism that causes melanoma cells to develop resistance to PLX4032-induced killing. When combining PLX4032 and the BCL-XL-specific inhibitor, A-1155463, HGF-mediated resistance can partially be overcome

showed that induction of PUMA by PLX4032 is independent on TP53 activation. These results are highly significant as they show that tumours that harbour mutations in p53, or have other defects in the p53 pathway, should still be able to upregulate PUMA and respond to PLX4032 treatment.

The question remains how PUMA is upregulated in BRAF $^{\mathrm{V} 600 E}$ melanoma cells in response to PLX4032 and how its upregulation is prevented by HGF/CMET signalling. We found that PUMA is mainly induced at the protein rather than at the mRNA level. This suggests that posttranslational modifications in PUMA may have an important role in PLX4032induced apoptosis. Along this line, it has been shown that PUMA phosphorylation primes this protein for proteasomal degradation. ${ }^{34}$ Future experiments are needed to investigate which component(s) of the PI3K or MAPK signalling pathways, driven by mutant BRAF and/or HGF/cMET signalling, cause PUMA phosphorylation and degradation.

Controversy surrounds the dependency of melanoma cells on different pro-survival BCL-2 family members for their sustained survival and growth. Although some report that $\mathrm{BCL}-2$ is important for cell survival during the progression to malignant melanoma, ${ }^{25}$ others reported that its expression is decreased in advanced melanomas compared with benign nevi. ${ }^{26}$ In accordance with the latter reports, we found that treatment with the BCL-2-specific inhibitor, ABT-199, did not increase PLX4032-induced apoptosis in any of the BRAF mutant cell lines, although they had detectable BCL-2 protein levels. BCL-XL was also reported to be highly expressed in metastatic melanoma. ${ }^{25}$ We found that inhibition of BCL-XL by the highly specific $\mathrm{BH} 3$ mimetic, A-1155463, did not promote apoptosis of BRAF mutant melanoma cells on its own, but greatly augmented apoptosis when combined with PLX4032. Hence, our study is the first to demonstrate that the synergistic pro-apoptotic response exerted by the combination of PLX4032 in combination with ABT-737, ${ }^{7,14}$ does not depend on inhibition of BCL-2 but on neutralization of BCL-XL. The finding that inhibition of $B C L-X L$ on its own is not sufficient to induce apoptosis is probably due to the fact that the low basal levels of pro-apoptotic BH3-only proteins present in the melanoma cells are not sufficient to cause BAX/BAK activation, either directly or indirectly by blocking pro-survival MCL-1, which is also present at readily detectable levels in these cells. Therefore, PLX4032-induced upregulation of BIM and PUMA is required to reach a threshold of BH3-only proteins sufficient to block all pro-survival BCL-2-like proteins and induce apoptosis. Indeed, when BIM was overexpressed in the BRAF mutant patient cell line that was less sensitive to PLX4032 (LM-MEL-33), very potent killing was observed 
(Figure 3b) compared with the levels of cell death seen following PLX4302 treatment. Although no clinical trials have yet been conducted with a BCL-XL selective inhibitor, our results provide evidence that certain melanomas are highly dependent on this pro-survival protein and that the combination of PLX4032 and BCL-XL inhibition could represent an attractive strategy for treatment of such cancers.

Several studies have demonstrated that activation of receptor tyrosine kinases by their corresponding growth factors can render PLX4032-sensitive melanoma cells resistant to apoptosis. ${ }^{16,17}$ In accordance with these studies, we found that HGF could rescue cMET+ BRAF mutant melanoma cell lines from PLX4032-induced killing, whereas those melanoma lines lacking cMET did not acquire resistance. Importantly, we were able to demonstrate that resistance to PLX4032 caused by HGF was associated with a reduction in PUMA and BIM protein levels rather than an increase in the levels of pro-survival BCL-2-like proteins.

Resistance of melanoma cells to PLX4032 has been proposed to be associated with selection for HGF-expressing tumour cells, ${ }^{17}$ and inhibition of cMET was therefore proposed as a strategy to promote killing of melanoma cells with high cMET levels. ${ }^{35,36}$ The small molecule drug SU11274 potently inhibits cMET tyrosine kinase activity, and this was shown to significantly reduce melanoma burden in a murine xenotransplantation model. ${ }^{37}$ Importantly, acquired resistance to SU11274 was observed and this was associated with upregulation of PI3K/AKT and WNT pathway signalling. Therefore, combination therapies with cMET, PI3K and WNT pathway inhibitors have been proposed as a novel strategy to treat such treatment refractory melanomas. ${ }^{37}$

In conclusion, we believe that in order to develop effective treatment strategies for melanoma patients, common mediators of resistance to PLX4032 need to be identified and targeted with suitable drugs. Signalling through PI3K/AKT and MAPK pathways by either growth factor-mediated activation of receptor tyrosine kinases or constitutively active mutants of RAS or BRAF all appear to affect the levels and activity of PUMA and BIM. If the downregulation of these two proapoptotic proteins is the most common mechanism for acquired drug resistance, then targeted therapies that keep PUMA and BIM levels high in cancer cells, or replacing their action by suitable $\mathrm{BH} 3$ mimetics that inhibit relevant prosurvival BCL-2 family members (BCL-XL and possibly also MCL-1 and BFL-1) may be of substantial benefit (Figure $6 \mathrm{~b}$ ).

\section{Materials and Methods}

Cell lines and compounds. The cell lines M14, UACC257, Malme3M, SKMEL5 and UACC62 were purchased from the ATCC (Manassas, VA, USA). Patient-derived cell lines LM-MEL-28, -33, -34 were obtained from the ONJCRI (Heidelberg, VIC, Australia). ${ }^{22} \mathrm{CHL}-1$ cells were obtained from the Peter MacCallum Cancer Institute, Melbourne, Australia. Drugs and growth factors were purchased or gifted to us as indicated: HGF (Sigma-Aldrich, St. Louis, MO, USA), PLX4032/Nemurafenib (Active Biochemicals, Wan Chai, Hong Kong, China), ABT-737, ABT-199 and A-1155463 (AbbVie Inc., North Chicago, IL, USA), Q-VDOPh (MP Biomedicals, Solon, OH, USA), Nutlin3a (Cayman Chemical, Ann Arbor, MI, USA).

Cell culture and generation of knockout cell lines using CRISPR/ Cas9. Cell lines were cultured in HT-RPMI 1640 culture medium supplemented with $5 \%$ foetal bovine serum (both from Thermo Fisher Scientific, Waltham, MA,
USA). For lentiviral particle production, 293T cells were transiently transfected with the packaging constructs PMDL $(5 \mu \mathrm{g})$, RSV-REV $(2.5 \mu \mathrm{g})$ and VSVg $(3 \mu \mathrm{g})$ along with $10 \mu \mathrm{g}$ vector DNA encoding Cas9 or individual sgRNAs. Cells were transfected using the calcium phosphate precipitation method as described previously. ${ }^{38}$ The generation of the Cas9 expression vector and sgRNA constructs was described recently. ${ }^{20}$ The sgRNA sequences are as follows:

- human BIM exon 2: 5'-GCCTCCCCAGCTCAGACCTG-3'

- human BIM exon 3: 5'-GCCCAAGAGTTGCGGCGTAT-3'

- human PUMA exon 2: 5'-AGCTCCCCGGAGCCCGTAGA-3'

- human PUMA exon 3: 5'-GCCGCTCGTACTGTGCGTTG-3'

- murine Bim exon 3: 5'-GCACAGGAGCTGCGGCGGAT-3

- human TP53 exon 4: 5'-GGCAGCTACGGTTTCCGTCT-3'

- human TP53 exon 5: 5'-GAGCGCTGCTCAGATAGCGA-3'.

To generate control or single knockout cell lines, Cas9-expressing cells $\left(1 \times 10^{4}\right.$ cells per well/six-well plate) were transduced with $500 \mu$ l lentivirus encoding human BIM or PUMA targeting sgRNAs or a murine Bim targeting sgRNA, as a negative control, in combination with $500 \mu$ l lentivirus encoding the empty sgRNA vector backbone. To generate double knockout cell lines, $500 \mu$ l human BIM sgRNA virus and $500 \mu \mathrm{l}$ human PUMA sgRNA virus were mixed and added together to target cells. Cells were cultured in the presence of virus for 3 days before culture medium was replaced. Flow cytometry was used to determine the percentage of mCherry (Cas9) and GFP (sgRNA) double-positive cells. When the transduction efficiency was lower than $95 \%$, then the double-positive cells were sorted as a whole population. To induce expression of the sgRNA, doxycycline-hyclate (Sigma-Aldrich) was added to the culture medium at a final concentration of $1 \mu \mathrm{g} / \mathrm{ml}$. Successful target gene disruption was verified by DNA sequence analysis as described previously. ${ }^{20}$

Inducible $\mathrm{BIM}_{\mathrm{S}}$ overexpression variants in patient melanoma cell lines. Patient-derived cell lines LM-MEL-28, -33, -34 (ref. 22) were transduced with doxycycline-inducible lentiviruses harbouring constructs encoding $\mathrm{BIM}_{\mathrm{S}}$ or the inert mutant, $B I M_{s} 4 E$, whose expression was linked to that of GFP via a T2A sequence. ${ }^{24}$ GFP-positive cells were sorted before analysis in killing assays. To induce expression of the ligands, cells were grown in the presence of doxycycline $(1 \mu \mathrm{g} / \mathrm{ml})$.

Cell survival assays and flow cytometric analysis. For survival assays, cells were seeded in 96-well flat-bottom tissue culture plates at 1000 cells per well in $100 \mu \mathrm{l}$ culture medium for established cell lines, and in 24-well flatbottom tissue culture plates at 30000 cells pe well in $500 \mu$ l culture medium for the low passage number human patient-derived melanoma cells. The next day, $100 \mu \mathrm{l}$ of $2 X$ concentrated compound-containing medium (PLX4032, HGF, ABT-737, ABT-199, A-1155463, doxycycline) or appropriate vehicle controls were added to the wells and cell survival was assessed by flow cytometric analysis. Non-transduced cells were resuspended in Annexin V binding buffer (0.1 M Hepes ( $\mathrm{pH} 7.4), 1.4 \mathrm{M}$ $\mathrm{NaCl}, 25 \mathrm{mM} \mathrm{CaCl}$ ) containing $5 \mu \mathrm{g} / \mathrm{ml}$ propidium iodide (PI) (Sigma-Aldrich) and Alexa Fluor 647-conjugated Annexin V (generated in house). Apoptosis of CRISPR/ Cas9 knockout cell lines was measured 5 days post-treatment by resuspending cells in Annexin V binding buffer containing Alexa Fluor 647-conjugated Annexin V. Survival of $\mathrm{BIM}_{\mathrm{S}}$ variant transduced patient melanoma cell lines was measured 3 days post-induction with doxycycline, using PI staining.

To measure cMET expression, cells were incubated for 30 min with biotinylated cMETantibody (eBioscience, San Diego, CA, USA), followed by incubation with FITCconjugated streptavidin (Thermo Fisher Scientific) for $30 \mathrm{~min}$. To measure the levels of pERK, approximately $1 \times 10^{4}$ cells were pelleted and resuspended in $200 \mu \mathrm{l}$ PFA $(1.5 \%)$ and incubated in fixative for $15 \mathrm{~min}$ at room temperature. Cells were pelleted and permeabilised by vigorous vortexing in $200 \mu \mathrm{l}$ ice-cold $\mathrm{MeOH}$ before incubating cells on ice for $20 \mathrm{~min}$. Cells were washed twice in staining medium (MT-PBS containing $1 \% \mathrm{BSA}$ ) and were then stained for 30 min with an APC-conjugated antibody against $p E R K$ (eBioscience). Samples were washed twice before analysis by flow cytometry in a LSRII machine (Becton Dickinson, Franklin Lakes, NJ, USA).

Western blot and qRT-PCR analyses. Cells were resuspended in $1 \mathrm{X}$ Onyx lysis buffer supplemented with protease inhibitors (Roche, Basel, Switzerland). Antibodies (clone) were purchased or obtained from the sources indicated: anti-BIM (Enzo Life Sciences, Farmingdale, NY, USA, cat\# ADI-AAP-330-E), antiPUMA (Abcam, Cambridge, UK, cat\# 27669), anti-BMF (WEHI, clone 12E10), antiNOXA (ProSci, Poway, CA, USA, cat\# 2437), anti-MCL-1 (WEHI, clone 19C4-15), anti-BCL-2 (WEHI, clone Bcl-2-100), anti-BCL-XL (WEHI, clone 9C9), anti-pERK (Cell Signaling, Danvers, MA, USA, cat\# 4370), anti-ERK (Cell Signaling, cat\# 
9102), anti-HSP70 (gift from Dr. R Anderson PMCC Melbourne, clone N6), antiTP53 (Santa Cruz, Dallas, TX, USA, cat\# 6243).

For qRT-PCR analysis, cells were resuspended in TRlzol reagent (Life Technologies, Carlsbad, CA, USA) and RNA was isolated following the manufacturer's protocol. RNA was reverse transcribed into cDNA utilizing the Super Script III First Strand cDNA Synthesis Kit (Thermo Fisher Scientific). The qPCR amplification was performed on an ABI 7900 machine (Applied Biosystems, Foster City, CA, USA) under standard running conditions. Each reaction was run in triplicate and data were analyzed using the $2 \Delta \Delta \mathrm{Ct}$ method where Gapdh was assigned as the housekeeping gene. All qRT-PCR primers were purchased from Life Technologies. The primers with the best coverage were chosen.

Statistical analysis. Experiments were analyzed using GraphPad PRISM version 6.0d (La Jolla, CA, USA) and Excel (Microsoft 2011). Statistical differences between sample groups were calculated using unpaired two-tailed Student's $t$-test. $P$-values of $<0.05$ were considered statistically significant.

\section{Conflict of Interest}

The authors declare no conflict of interest.

Acknowledgements. We thank all members of the MJ Herold laboratory as well as Drs. ARD Delbridge, B Aubrey, S Grabow and Ms. T Harris for their support and advice; Drs. JM Adams and ARD Delbridge for comments on the article; Drs. L O'Connor, L Milla and S Wilcox for help with the interpretation of sequencing results; and Dr. S Monard and his team for help with flow cytometry. This work was supported by the Melbourne International Research Scholarship and the Melbourne International Fee Remission Scholarship (to LR), the Australian Cancer Research Foundation, the National Health and Medical Research Council, Australia program grant 1016701 and fellowship 1020363, Leukemia and Lymphoma Society SCOR grant 7001-13 (to AS) and grants 7015 and 7413 (to GL), project grant GNT1049720 (to MJH) and GNT1025138 (to GL) a Worldwide Cancer Research Grant 15-0025 (to WDF, MJH, EFL, AS) and Australian Research Council Future Fellowship FR150100212 (to EFL). This work was made possible through Victoria State Government Operational Infrastructure Support and Australian Government National Health and Medical Research Council Independent Research Institutes Infrastructure Support Scheme.

1. Wan PT, Garnett MJ, Roe SM, Lee S, Niculescu-Duvaz D, Good VM et al. Mechanism of activation of the RAF-ERK signaling pathway by oncogenic mutations of B-RAF. Cell 2004; 116: 855-867.

2. Davies H, Bignell GR, Cox C, Stephens $\mathrm{P}$, Edkins $\mathrm{S}$, Clegg $\mathrm{S}$ et al. Mutations of the BRAF gene in human cancer. Nature 2002; 417: 949-954.

3. Cartlidge RA, Thomas GR, Cagnol S, Jong KA, Molton SA, Finch AJ et al. Oncogenic BRAF (V600E) inhibits BIM expression to promote melanoma cell survival. Pigment Cell Melanoma Res 2008; 21: 534-544.

4. O'Reilly LA, Kruse EA, Puthalakath H, Kelly PN, Kaufmann T, Huang DC et al. MEK/ERKmediated phosphorylation of Bim is required to ensure survival of $T$ and $B$ lymphocytes during mitogenic stimulation. J Immunol 2009; 183: 261-269.

5. Tsai J, Lee JT, Wang W, Zhang J, Cho H, Mamo S et al. Discovery of a selective inhibitor of oncogenic B-Raf kinase with potent antimelanoma activity. Proc Natl Acad Sci USA 2008; 105: 3041-3046.

6. Cragg MS, Jansen ES, Cook M, Strasser A, Scott CL. Treatment of B-RAF mutant human tumor cells with a MEK inhibitor requires Bim and is enhanced by a BH3 mimetic. $J$ Clin Invest 2008; 118: 3651-3659.

7. Serasinghe MN, Missert DJ, Asciolla JJ, Podgrabinska S, Wieder SY, Izadmehr S et al. Antiapoptotic BCL-2 proteins govern cellular outcome following B-RAF inhibition and can be targeted to reduce resistance. Oncogene 2014; 34: 857-867.

8. Merino D, Giam M, Hughes PD, Siggs OM, Heger K, O'Reilly LA et al. The role of BH3-only protein Bim extends beyond inhibiting Bcl-2-like prosurvival proteins. J Cell Biol 2009; 186: 355-362.

9. Llambi F, Moldoveanu T, Tait SW, Bouchier-Hayes L, Temirov J, McCormick LL et al. A unified model of mammalian BCL-2 protein family interactions at the mitochondria. Mol Cell 2011; 44: 517-531.

10. Czabotar PE, Lessene G, Strasser A, Adams JM. Control of apoptosis by the BCL-2 protein family: implications for physiology and therapy. Nat Rev Mol Cell Biol 2014; 15 : 49-63.
11. Oltersdorf T, Elmore SW, Shoemaker AR, Armstrong RC, Augeri DJ, Belli BA et al. An inhibitor of Bcl-2 family proteins induces regression of solid tumours. Nature 2005; 435 : 677-681.

12. Kuroda J, Puthalakath H, Cragg MS, Kelly PN, Bouillet P, Huang DC et al. Bim and Bad mediate imatinib-induced killing of Bcr/Abl+ leukemic cells, and resistance due to their loss is overcome by a BH3 mimetic. Proc Natl Acad Sci USA 2006; 103: 14907-14912.

13. Cragg MS, Kuroda J, Puthalakath H, Huang DCS, Strasser A. Gefitinib-induced killing of NSCLC cell lines expressing mutant EGFR requires Bim and can be enhanced by $\mathrm{BH} 3$ mimetics. PLoS Med 2007; 4: 1681-1689.

14. Wroblewski D, Mijatov B, Mohana-Kumaran N, Lai F, Gallagher SJ, Haass NK et al. The BH3mimetic ABT-737 sensitizes human melanoma cells to apoptosis induced by selective BRAF inhibitors but does not reverse acquired resistance. Carcinogenesis 2013; 34: 237-247.

15. Flaherty KT, Puzanov I, Kim KB, Ribas A, McArthur GA, Sosman JA et al. Inhibition of mutated, activated BRAF in metastatic melanoma. N Engl J Med 2010; 363: 809-819.

16. Wilson TR, Fridlyand J, Yan Y, Penuel E, Burton L, Chan E et al. Widespread potential for growth-factor-driven resistance to anticancer kinase inhibitors. Nature 2012; 487: 505-509.

17. Straussman R, Morikawa T, Shee K, Barzily-Rokni M, Qian ZR, Du J et al. Tumour microenvironment elicits innate resistance to RAF inhibitors through HGF secretion. Nature 2012; 487: 500-504.

18. Yu J, Zhang L, Hwang PM, Kinzler KW, Vogelstein B. PUMA induces the rapid apoptosis of colorectal cancer cells. Mol Cell 2001; 7: 673-682.

19. Nakano K, Vousden KH. PUMA, a novel proapoptotic gene, is induced by p53. Mol Cell 2001; 7: 683-694.

20. Aubrey BJ, Kelly GL, Kueh AJ, Brennan MS, O'Connor L, Milla L et al. An inducible lentiviral guide RNA platform enables the identification of tumor-essential genes and tumor-promoting mutations in vivo. Cell Rep 2015; 10: 1422-1432.

21. Valente LJ, Aubrey BJ, Herold MJ, Kelly GL, Happo L, Newbold A et al. Therapeutic response to non-genotoxic activation of p53 by Nutlin3a is driven by PUMA-mediated apoptosis in lymphoma cells. Cell Rep 2016; 14: 1858-1866.

22. Behren A, Anaka M, Lo PH, Vella LJ, Davis ID, Catimel J et al. The Ludwig institute for cancer research Melbourne melanoma cell line panel. Pigment Cell Melanoma Res 2013; 26: 597-600.

23. Kelly GL, Grabow S, Glaser SP, Fitzsimmons L, Aubrey BJ, Okamoto T et al. Targeting of MCL-1 kills MYC-driven mouse and human lymphomas even when they bear mutations in p53. Genes Dev 2014; 28: 58-70.

24. Chen L, Willis SN, Wei A, Smith BJ, Fletcher Jl, Hinds MG et al. Differential targeting of pro-

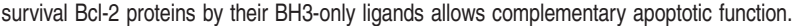
Mol Cell 2005; 17: 393-403.

25. Leiter U, Schmid RM, Kaskel P, Peter RU, Krahn G. Antiapoptotic bcl-2 and bcl-xL in advanced malignant melanoma. Arch Dermatol Res 2000; 292: 225-232.

26. Zhuang L, Lee CS, Scolyer RA, McCarthy SW, Zhang XD, Thompson JF et al. Mcl-1, Bcl-XL and Stat3 expression are associated with progression of melanoma whereas Bcl-2, AP-2 and MITF levels decrease during progression of melanoma. Mod Pathol 2007; 20: 416-426.

27. Haq R, Yokoyama S, Hawryluk EB, Jonsson GB, Frederick DT, McHenry K et al. BCL2A1 is a lineage-specific antiapoptotic melanoma oncogene that confers resistance to BRAF inhibition. Proc Natl Acad Sci USA 2013; 110: 4321-4326.

28. Souers AJ, Leverson JD, Boghaert ER, Ackler SL, Catron ND, Chen J et al. ABT-199, a potent and selective BCL-2 inhibitor, achieves antitumor activity while sparing platelets. Nat Med 2013; 19: 202-208.

29. Tao ZF, Hasvold L, Wang L, Wang X, Petros AM, Park CH et al. Discovery of a potent and selective BCL-XL inhibitor with in vivo activity. ACS Med Chem Lett 2014; 5: 1088-1093.

30. Jubb AM, Ribas A, Sosman JA, McArthur GA, Yan Y, Rost S et al. Impact of MET expression on outcome in BRAF(V600E/K) advanced melanoma. Histopathology 2013; 63: 351-361.

31. Jeffers JR, Parganas E, Lee $Y$, Yang $C$, Wang J, Brennan J et al. Puma is an essential mediator of p53-dependent and -independent apoptotic pathways. Cancer Cell 2003; 4: 321-328.

32. Villunger A, Michalak EM, Coultas L, Mullauer F, Bock G, Ausserlechner MJ et al. p53- and drug-induced apoptotic responses mediated by $\mathrm{BH}$-only proteins puma and noxa. Science 2003; 302: 1036-1038

33. Erlacher M, Michalak EM, Kelly PN, Labi V, Niederegger H, Coultas L et al. BH3-only proteins Puma and Bim are rate-limiting for \{gamma\} -radiation and glucocorticoid-induced apoptosis of lymphoid cells in vivo. Blood 2005; 106: 4131-4138.

34. Sandow JJ, Jabbour AM, Condina MR, Daunt CP, Stomski FC, Green BD et al. Cytokine receptor signaling activates an IKK-dependent phosphorylation of PUMA to prevent cell death. Cell Death Differ 2012; 19: 633-641.

35. Puri N, Ahmed S, Janamanchi V, Tretiakova M, Zumba O, Krausz T et al. c-Met is a potentially new therapeutic target for treatment of human melanoma. Clin Cancer Res 2007: 13: 2246-2253.

36. Sattler M, Salgia R. c-Met and hepatocyte growth factor: potential as novel targets in cancer therapy. Curr Oncol Rep 2007; 9: 102-108.

37. Etnyre D, Stone AL, Fong JT, Jacobs RJ, Uppada SB, Botting GM et al. Targeting c-Met in melanoma: mechanism of resistance and efficacy of novel combinatorial inhibitor therapy. Cancer Biol Ther 2014; 15: 1129-1141.

38. Herold MJ, van den Brandt J, Seibler J, Reichardt HM. Inducible and reversible gene silencing by stable integration of an shRNA-encoding lentivirus in transgenic rats. Proc Natl Acad Sci USA 2008; 105: 18507-18512. 